\title{
ON A PROBLEM OF R. G. D. RICHARDSON
}

\author{
by PAUL BINDING and HANS VOLKMER
}

(Received 12th March 1990)

In 1913 Richardson published necessary and sufficient conditions for a system of three Sturm-Liouville equations, linked by three parameters, to possess eigenfunctions with arbitrarily many zeros. His work contains errors, but we give conditions of his type valid for $k$ self-adjoint equations, with $k$ parameters.

1980 Mathematics subject classification (1985 Revision): $34 B 25$

\section{Introduction}

In 1912 R. G. D. Richardson published a landmark paper [12] on coupled Sturm-Liouville problems of the form

$$
-\left(p_{m} y_{m}^{\prime}\right)^{\prime}+q_{m} y_{m}=\sum_{n=1}^{k} \lambda_{n} r_{m n} y_{m} \quad 1 \leqq m \leqq k
$$

for $k=2$. Richardson assumed Dirichlet end conditions on finite intervals, and analytic coefficient functions $p_{n}>0, q_{m}$ and $r_{m n}$, and he obtained necessary and sufficient conditions on the $r_{m n}$ guaranteeing existence of solutions with specified numbers of zeros for the eigenfunctions $y_{m}$. A year later he attempted [13] the more difficult case $k=3$. His methods involved detailed examination of eigencurves and surfaces defined by single equations in two and three parameters, and led to a unified oscillation theory for indefinite, as well as the better known left ("polar") and right definite cases. In many ways these investigations were far ahead of their time: for example, oscillation theory for indefinite problems really restarted in the early 1970's (cf. [6], [7] etc.) and a systematic study of the geometry of eigencurves and surfaces resurfaced with Turyn [14] in 1979, although specific properties of eigencurves can be found in, say, [10], [1], [9] and [8].

Richardson's geometric reasoning can easily be put into a more modern (and rigorous) analytical framework when $k=2$, but there are difficulties when $k=3$, as observed by Turyn (loc. cit). In fact we shall show that Richardson's necessary and sufficient conditions in [13] are both in error, but we shall examine special cases for $k=3$ where his reasoning does lead to correct conclusions. Our general results will be couched in terms of self-adjoint operators acting on $k$ Hulbert spaces $H_{m}, m=1, \ldots, k$, and the Sturm-Liouville setting (1.1) will be deduced as a special case. Necessary and sufficient conditions for the existence of eigenvalues can also be found in [15] and 
relevant references therein, but under special "definiteness" conditions. Our methods here are rather different, since, following Richardson, we presuppose no definiteness conditions.

Our plan is as follows. In Section 2 we shall discuss a general necessary condition and compare it with "left" and "right" definiteness, In Section 3 we specialise this to the Sturm-Liouville case and we compare it with Richardson's $k=3$ condition, which we show is not in fact necessary. In Section 4 we establish a sufficient condition by a degree theoretic argument. Finally Section 5 is devoted to comparisons between our sufficient conditions, our necessary conditions, left definiteness (which although related is not directly comparable with our conditions) and Richardson's sufficient conditions. We conclude with an example to show that his $k=3$ condition is not in fact sufficient.

\section{A necessary condition}

Let $T_{m}, V_{m n}, n=1, \ldots, k$, be self-adjoint operators on separable Hilbert spaces $H_{m}, T_{m}$ being bounded below with compact resolvents and $V_{m n}$ being bounded, and let $W_{m}(\lambda)=T_{m}-V_{m}(\lambda)$, where $V_{m}(\lambda)=\sum_{n=1}^{k} V_{m n} \lambda_{n}, m=1, \ldots, k$. Here and below we assume $\lambda=\left[\lambda_{1} \ldots \lambda_{k}\right]^{T} \in \mathbb{R}^{k}$. Let the eigenvalues of $W_{m}(\lambda)$ be listed as

$$
\rho_{m}^{1}(\lambda) \leqq \rho_{m}^{2}(\lambda) \leqq \ldots,
$$

counted by multiplicity. With $U_{m}$ as the unit sphere of $H_{m}$, the multiparameter eigenvalue problem

$$
W_{m}(\lambda) u_{m}=0, \quad u_{m} \in U_{m} \cap D\left(T_{m}\right), \quad m=1, \ldots, k,
$$

can be studied via the equations

$$
\rho_{m}^{i_{m}}(\lambda)=0, \quad m=1, \ldots, k .
$$

Indeed any solution of (2.2) yields nontrivial nullspaces for each $W_{m}(\lambda)$, and conversely any $\lambda$ satisfying (2.1) also satisfies (2.2) for some index $\mathbf{i}=\left(i_{1}, \ldots, i_{k}\right)$. For examples of this setting, we refer, for instance, to [15].

We use lower case letters to denote quadratic forms, e.g., $t_{m}(u)=\left(u, T_{m} u\right)$, and $V(u)$ will be the $k \times k$ matrix with $(m, n)$ entry $v_{m n}\left(u_{m}\right)$ and with $m$ th row $v_{m}\left(u_{m}\right)$. Our study of the equations (2.2) depends mainly on the following representation of the functions $\rho_{m}^{i_{m}}$ given by the minimax principle

$$
\rho_{m}^{i_{m}}(\lambda)=\min \left\{\max \left\{\mathrm{t}_{\mathrm{m}}(\mathrm{u})-\mathrm{v}_{\mathrm{m}}(\mathrm{u}) \lambda: u \in U_{m} \cap E\right\}: E \subset D\left(T_{m}\right), \operatorname{dim} E=i_{m}\right\} .
$$

For any subspace $G$ of $H_{m}$, we write

$$
l_{m}(\lambda, G)=\inf \left\{\mathbf{v}_{m}(u) \lambda: u \in U_{m} \cap G\right\} .
$$


It is convenient to make the assumption

(A) For each $m$ and $\lambda$, the maximum of the spectrum of $V_{m}(\lambda)$ is not an eigenvalue of finite multiplicity.

Then we define

$$
\sigma_{m}(\lambda)=\sup \left\{l_{m}(\lambda, G): G \subset D\left(T_{m}\right), \operatorname{dim} G=i\right\}
$$

for any fixed finite $i$ and by virtue of (A) we have

$$
\sigma_{m}(\lambda)=\sup \left\{\mathbf{v}_{m}(u) \lambda: u \in U_{m}\right\}
$$

We shall need three lemmas involving single equations, i.e. with $m$ fixed.

Lemma 2.1. Let $i=i_{m}$ be fixed and let the sequence $\lambda^{j} \in \mathbb{R}^{k}$ satisfy $\rho_{m}^{i}\left(\lambda^{j}\right) \geqq 0$ for all $j$, with $\left\|\lambda^{j}\right\| \rightarrow \infty$ and $\lambda^{j} /\left\|\lambda^{j}\right\| \rightarrow \lambda^{*}$. Then $\sigma_{m}\left(\lambda^{*}\right) \leqq 0$.

Proof. For any $i$ dimensional subspace $F$ of $D\left(T_{m}\right),(2.3)$ gives

$$
t_{m}\left(u^{j}\right)-\mathbf{v}_{m}\left(u^{j}\right) \lambda^{j} \geqq 0
$$

for some $u^{j} \in U_{m} \cap F$. Assuming, without loss of generality, that $u^{j} \rightarrow u \in U_{m} \cap F$, we may divide (2.5) by $\left\|\lambda^{j}\right\|$ and proceed to the limit to give

$$
\mathbf{v}_{m}(u) \lambda^{*} \leqq 0
$$

for some $u \in U_{m} \cap F$. By definition of $\sigma_{m}\left(\lambda^{*}\right)$, this suffices for the result.

Lemma 2.2. As for Lemma 2.1, but with the inequalities reversed.

Proof. By assumption, $\rho_{m}^{1}(\lambda) \leqq \rho_{m}^{i}(\lambda) \leqq 0$. Then (2.3) gives (2.5) (with the inequality reversed) for some sequence $u^{j} \in U_{m} \cap D\left(T_{m}\right)$. We again divide by $\left\|\lambda^{j}\right\|$ and obtain

$$
\mathbf{v}_{m}\left(u^{j}\right) \lambda^{*} \geqq \frac{t_{m}\left(u^{j}\right)}{\left\|\lambda^{j}\right\|}-\mathbf{v}_{m}\left(u^{j}\right)\left(\frac{\lambda^{j}}{\left\|\lambda^{j}\right\|}-\lambda^{*}\right)
$$

Since $t_{m}\left(u^{j}\right)$ is bounded below, we see that, for every given $\varepsilon>0, \mathbf{v}_{m}\left(u^{j}\right) \lambda^{*}>-\varepsilon$ whenever $j$ is sufficiently large. The result now follows from (2.4).

Lemma 2.3. Let $i(j)$ be a sequence of positive integers converging to infinity and let $\rho_{m}^{i(j)}\left(\lambda^{j}\right)=0$ for all $j$. Then $\left\|\lambda^{j}\right\| \rightarrow \infty$.

Proof. For each $i, \rho_{m}^{i}\left(\lambda^{j}\right) \leqq 0$ as $j \rightarrow \infty$. Thus if $\lambda^{j}$ has an accumulation point $\lambda^{*}$ in $\mathbb{R}^{k}$, 
then, by continuity of $\rho_{m}^{i}$, it follows that $\rho_{m}^{i}\left(\lambda^{*}\right) \leqq 0$ for all $i$. This contradicts the fact that $\rho_{m}^{i}\left(\lambda^{*}\right) \rightarrow \infty$ as $i \rightarrow \infty$.

We are now ready for the basic necessary condition.

Theorem 2.4. Fix $m$ and positive integers $i_{n}$ for $n=1, \ldots, k, n \neq m$. If, for an infinite set of $i_{m},(2.2)$ admits eigenvalues $\lambda=\lambda^{(m)}$, then there is $\mu \neq 0$ such that

$$
\sigma_{n}(\mu)=0 \leqq \sigma_{m}(\mu)
$$

if $n=1, \ldots, k, n \neq m$.

Proof. By Lemma 2.3, the eigenvalues $\lambda^{(m)}$ satisfy $\left\|\lambda^{(m)}\right\| \rightarrow \infty$ as $i_{m} \rightarrow \infty$. Let $\boldsymbol{\mu}$ be an accumulation point of the sequence $\lambda^{(m)} /\left\|\lambda^{(m)}\right\|$. Then $\sigma_{n}(\mu)=0$ follows readily from Lemmas 2.1 and 2.2. Moreover $\rho_{m}^{1}\left(\lambda^{(m)}\right) \leqq \rho_{m}^{i_{m}}\left(\lambda^{(m)}\right)=0$ so Lemma 2.2 gives $0 \leqq \sigma_{m}(\mu)$.

Corollary 2.5. If there is a fixed index $\mathbf{i}$ so that (2.2) admits eigenvalues of any index $\mathbf{j} \geqq \mathbf{i}$ then for any nonempty subset $\Sigma$ of $\{1, \ldots, k\}$ there is $\mu^{\Sigma} \neq \mathbf{0}$ so that (2.6) holds for $\boldsymbol{\mu}=\boldsymbol{\mu}^{\Sigma}$ if $\boldsymbol{m \in \Sigma}$ and $n \notin \Sigma$.

Here and below we use the componentwise order on $\mathbb{R}^{k}$.

\section{Differential equations}

With reference to (1.1), suppose $1 / p_{m}$ and $q_{m}$ are $L_{1}$ with $p_{m}$ positive, and define

$$
D\left(T_{m}\right)=\left\{y \in C^{1}\left[a_{m}, b_{m}\right] \cap B C: p_{m} y^{\prime} \in A C\left[a_{m}, b_{m}\right],-\left(p_{m} y^{\prime}\right)^{\prime}+q_{m} y \in L_{2}\left[a_{m}, b_{m}\right]\right\}
$$

where $B C$ represents separated boundary conditions. Then define

$$
T_{m} y=-\left(p_{m} y^{\prime}\right)^{\prime}+q_{m} y,\left(V_{m n} y\right)\left(x_{m}\right)=r_{m n}\left(x_{m}\right) y\left(x_{m}\right)
$$

so $[11, \S 18,19]$ the hypotheses placed on $T_{m}$ and $V_{m n}$ in Section 2 are satisfied provided the $r_{m n}$ are $L_{\infty}$.

It is well known (cf. [15, Theorem 3.5.1]) that a solution of (2.1) has index $\mathbf{i}$ if and only if the corresponding solution $y_{m}$ of (1.1) has $i_{m}-1$ zeros in $] a_{m}, b_{m}[$. This leads us to the following condition which is of Richardson's type, and which is a special case of Corollary 2.5. By virtue of (2.4), the functions $\sigma_{m}$ now become

$$
\sigma_{m}(\lambda)=\operatorname{ess} \sup \left\{\sum_{n=1}^{k} \lambda_{n} r_{m n}\left(x_{m}\right): a_{m} \leqq x_{m} \leqq b_{m}\right\}
$$

Corollary 3.1. If (1.l) admits solutions with abritrarily many zeros for each $y_{m}$, then there exist $\boldsymbol{\mu}^{m} \neq 0, m=1, \ldots, k$, such that, for all $m, n=1, \ldots, k$,

$$
\sigma_{n}\left(\mu^{m}\right)=0 \text { if } m \neq n
$$




$$
\sigma_{n}\left(\mu^{m}\right) \geqq 0 \quad \text { if } \quad m=n
$$

For comparison, we state Richardson's necessary condition (for $k=3$ and with analytic $\left.p_{m}, q_{m}, r_{m n}\right)$ in the following form. Let $R(x)$ be the $3 \times 3$ matrix with $(m, n)$ th entry $r_{m n}\left(x_{m}\right)$ in (1.1).

(RNC) There exists vectors $\xi^{m}\left(=-\left[\lambda^{(m)} \mu^{(m)} v^{(m)}\right]^{T}\right.$ in his notation) such that, for all $m, n=1,2,3$,

$$
\left(R(x) \xi^{m}\right)_{n} \leqq 0 \text { for all } x_{n} \text { if } m \neq n
$$

and

$$
\left(R(x) \xi^{m}\right)_{m}=0 \text { for some } x_{m}
$$

In our notation (2.4), (3.1) this means

$$
\sigma_{n}\left(\xi^{m}\right) \leqq 0 \quad \text { if } \quad m \neq n
$$

and

$$
l_{m}\left(\xi^{m}, U_{m}\right) \leqq 0 \leqq \sigma_{m}\left(\xi^{m}\right) \text { for all } m
$$

It is evident that our conditions in Corollary 3.1 imply RNC except for the left-hand inequality in (3.7), and the following example shows that this infimal condition is not necessary.

Example 3.2. Let $p_{m} \equiv 1, q_{m} \equiv 0$ for $m=1,2,3$, and let

$$
R(x)=\left[\begin{array}{ccc}
1-x_{1} & -x_{1} & 0 \\
-x_{2} & 1-x_{2} & 0 \\
0 & 0 & 1
\end{array}\right]
$$

We solve (1.1) over the interval $[0,1]$ with Dirichlet end conditions; since the coefficients are analytic, this is a problem of Richardson's type. We claim that solutions of (1.1) exist for each index $i \geqq(1,1,1)$. This is a consequence of Theorem 4.1 below, but may also be seen as follows.

For $m=3$, we have the (right definite) problem $-y_{3}^{\prime \prime}=\lambda_{3} y_{3}$, which evidently has solutions of any index $i_{3} \geqq 1$. For $m=1,2$ we have a left definite system $[15$, p. 43], since the cofactor matrix $C(x)$ of

$$
\left[\begin{array}{cc}
1-x_{1} & -x_{1} \\
-x_{2} & 1-x_{2}
\end{array}\right]
$$

satisfies

$$
C(x)\left[\begin{array}{l}
1 \\
1
\end{array}\right]=\left[\begin{array}{l}
1 \\
1
\end{array}\right],
$$


and $T_{m}$ is positive definite for $m=1,2$. Moreover the determinant of

$$
\left[\begin{array}{cc}
1-x_{1} & -x_{1} \\
-x_{2} & 1-x_{2}
\end{array}\right]
$$

is not identically zero. Thus solutions $\lambda_{1}, \lambda_{2}$ exist of any index $\left(i_{1}, i_{2}\right) \geqq(1,1)-$ see, e.g., [15, Theorem 3.5.2].

It follows that (3.2), (3.3) must hold: indeed it is enough to choose the $\mu^{m}$ as the unit coordinate vectors in $\mathbb{R}^{3}$. On the other hand, (3.4) requires

$$
\left[\begin{array}{ccc}
1-x_{1} & -x_{1} & 0 \\
-x_{2} & 1-x_{2} & 0
\end{array}\right] \xi^{3} \leqq\left[\begin{array}{l}
0 \\
0
\end{array}\right] \text { for all } x_{1}, x_{2} \in[0,1]
$$

and this forces $\xi^{3}=\left[\begin{array}{lll}0 & 0 & \alpha\end{array}\right]^{T}$. Further, (3.5) requires $\left[\begin{array}{lll}0 & 0 & 1\end{array}\right] \xi^{3}$ to vanish for some $x_{3}$, so $\alpha=0$ and thus RNC fails if one excludes the triviality $\xi^{3}=0$.

We conclude this section by comparing the above conditions with the standard ones of right definiteness (RD) and left definiteness (LD). RD means that $V(u)$ has determinant of one sign for all choices of the $u_{m}$. This implies that, for each subset $\Sigma$ (including $\emptyset$ ) of $\{1, \ldots, k\}$, there is $\boldsymbol{v}^{\boldsymbol{\Sigma}} \neq \mathbf{0}$ such that

$$
\sigma_{n}\left(v^{\Sigma}\right) \leqq 0 \leqq l_{m}\left(v^{\Sigma}, U_{m}\right)\left(\leqq \sigma_{m}\left(v^{\Sigma}\right)\right)
$$

for all $m \in \Sigma$ and $n \notin \Sigma$. See (2.4), (3.1) for the notation and [2, Theorem 9.7.1] for a stronger result. Since [15, Theorem 3.5.2] RD implies that eigenvalues exist for all indices $\mathrm{i}$, Corollary 2.5 shows that (2.6) must hold for some $\mu^{\Sigma}$ for all $\Sigma \neq \emptyset$. A direct proof that RD implies (2.6) is not obvious in general, but for $k \leqq 3$, simple geometric arguments based on perturbing $v^{\Sigma}$ will suffice. In similar fashion one can show that RD implies (3.7) and hence RNC.

Analogous reasoning holds for $\mathrm{LD}$, which consists of a definiteness condition on the $T_{m}$, and an "ellipticity" condition which requires existence of a unit $\omega \in \mathbb{R}^{k}$ such that $C(u) \omega>0$, where $C(u)$ is the cofactor matrix of $V(u)$. Rotating axes so that $\omega=$ $\left[\begin{array}{ll}0 \ldots 0 & 1\end{array}\right]^{T}$, we may use $[3$, Theorem 6.3$]$ to show that ellipticity implies the existence for each $\Sigma \neq \emptyset$ of $v^{\Sigma}$, with $v_{k}^{\Sigma}=0$, such that (3.8) holds. Thus the above implications of RD also hold for $\mathrm{LD}$.

\section{A sufficient condition}

The purpose of this section is to prove the following result for the operators $T_{m}$ and $V_{m n}$ of Section 2. We recall assumption (A) and the subsequent definition of $\sigma_{m}$.

Theorem 4.1. Suppose that

(i) the $i_{m}$ th eigenvalue of $T_{m}$, i.e. $\rho_{m}^{i_{m}}(0)$, os positive for $m=1, \ldots, k$.

(ii) $V_{m n} \leqq 0$ if $m, n=1, \ldots, k, m \neq n$. 
(iii) for some $\mu>0, \sigma_{m}(\mu)>0$ for all $m=1, \ldots, k$.

Then (2.1) has a solution $\lambda>0$ of any given index $\mathbf{j} \geqq \mathbf{i}$.

Remarks. (a) Theorem 4.1 applies to Example 3.2 with $\mu=\left[\begin{array}{lll}1 & 1 & 1\end{array}\right]^{T}$.

(b) By (iii), there are $i_{m}$-dimensional subspaces $E_{m}$ of $D\left(T_{m}\right)$ such that, for some $\mu>0$,

$$
V(u) \mu>0 \text { for all } u \in E:=\mathrm{X}_{m=1}^{k}\left(E_{m} \cap U_{m}\right) .
$$

We also note by (i) and the minimax principle (2.3) that there is $e \in E$ such that

$$
t_{m}\left(e_{m}\right) \geqq \rho_{m}^{i_{m}}(\mathbf{0})>0, \quad m=1, \ldots, k .
$$

(c) If we drop our assumption (A) on the spectra of the $V_{m}(\lambda)$ then Theorem 4.1 remains true if we replace assumption (iii) by (4.1).

We shall need two lemmas, the first involving the set

$$
D=\left\{\lambda \in \mathbb{R}^{k}: \lambda>0 \text { and } \mathbf{t}(u)>V(u)(\lambda-\mu) \text { for some } u \in E\right\}
$$

where $\mathbf{t}=\left[t_{1} \ldots t_{k}\right]^{T}$.

Lemma 4.2. $\quad D$ is a nonempty open bounded subset of $\mathbb{R}^{k}$.

Proof. Openness of $D$ is elementary. For any $u \in E$, (ii) and (4.1) give $v_{m n}\left(u_{m}\right) \leqq 0$ whenever $m \neq n$ and $V(u) \mu>0$, for some $\mu>0$. By a well known result, cf. [15, Lemma 5.5.1], $V(u)^{-1}$ exists with all elements nonnegative. In particular

$$
\lambda(e):=V(e)^{-1} \mathbf{t}(e) \in D .
$$

Moreover, for any $\lambda \in D$ there is $u \in E$ such that

$$
\mathbf{0}<\lambda<\boldsymbol{\mu}+V(u)^{-1} \mathbf{t}(u)
$$

These bounds are uniform because $V(u)^{-1} t(u)$ is continuous on the compact set $E$.

Our second lemma concerns the function $\mathbf{g}=[0,1] \times \mathbb{R}^{k} \rightarrow \mathbb{R}^{k}$ given by

$$
\begin{gathered}
g_{m}(\alpha, \lambda)=\inf \left\{\operatorname { m a x } \left\{t_{m}\left(u_{m}^{\alpha}\right)-v_{m}\left(u_{m}^{\alpha}\right) \lambda: u_{m}^{\alpha}=\alpha^{1 / 2} e_{m}+(1-\alpha)^{1 / 2} u_{m},\right.\right. \\
\left.\left.u_{m} \in U_{m} \cap F_{m}, \operatorname{Re}\left(e_{m}, u_{m}\right)=0\right\}: F_{m} \subset D\left(T_{m}\right), \operatorname{dim} F_{m}=i_{m}\right\}
\end{gathered}
$$

Standard arguments using the semiboundedness of $t_{m}\left(u_{m}\right)$ show that $\mathrm{g}$ is continuous.

Lemma 4.3. For each $\alpha \in[0,1]$, the map $\mathbf{g}(\alpha, \cdot)$ does not vanish on the boundary $\partial D$ of $D$.

Proof. Elementary considerations show that if $\lambda \in \partial D$ then for some $m$ either 
(a) $\lambda_{m}=0$

or

(b) $\max \left\{t_{m}\left(u_{m}\right)-v_{m}\left(u_{m}\right)(\lambda-\mu): u_{m} \in E_{m} \cap U_{m}\right\}=0$.

(a) For any $i_{m}$-dimensional subspace $F_{m}$ of $D\left(T_{m}\right)$, choose $u_{m} \in F_{m} \cap U_{m}$ so that $t_{m}\left(u_{m}\right) \geqq \rho_{m}^{i_{m}}(0), \operatorname{Re}\left(e_{m}, u_{m}\right)=0$ and $\operatorname{Re}\left(T_{m} e_{m}, u_{m}\right) \geqq 0-$ see (i). In the notation of (4.4), then, $t_{m}\left(u_{m}^{a}\right) \geqq \rho_{m}^{i_{m}}(0)$ by (4.2). Thus from (ii) and $\lambda_{m}=0$ we have

$$
t_{m}\left(u_{m}^{\alpha}\right)-\mathbf{v}_{m}\left(u_{m}^{\alpha}\right) \lambda \geqq \rho_{m}^{i_{m}}(\mathbf{0})
$$

It follows that $g_{m}(\alpha, \lambda) \geqq \rho_{m}^{i_{m}}(0)>0$.

(b) For any $u_{m} \in U_{m} \cap E_{m}$ such that $\operatorname{Re}\left(e_{m}, u_{m}\right)=0$, we have by (4.1) and (4.5)

$$
t_{m}\left(u_{m}^{\alpha}\right)-\mathbf{v}_{m}\left(u_{m}^{\alpha}\right) \lambda=t_{m}\left(u_{m}^{\alpha}\right)-\mathbf{v}_{m}\left(u_{m}^{\alpha}\right)(\lambda-\mu)-\mathbf{v}_{m}\left(u_{m}^{\alpha}\right) \mu<0
$$

and so $g_{m}(\alpha, \lambda)<0$.

We are now ready to prove Theorem 4.1. Since $V(e)$ is invertible (as above) and

$$
\mathbf{g}(1, \lambda)=\mathbf{t}(e)-V(e) \lambda
$$

vanishes only at $\lambda(e) \in D$ by (4.3) we have by Lemma 4.2 that the degree $\operatorname{deg}(g(\alpha, \cdot), D, 0)$ is well defined and nonzero for $\alpha=1$. By Lemma 4.3, this remains true for $\alpha=0$. Now (2.3) shows that

$$
g_{m}(0, \lambda)=\rho_{m}^{i_{m}}(\lambda)
$$

so there must be a solution to (2.2) in $D$. For $\mathbf{j} \geqq \mathbf{i}$ we note that (i)-(iii) hold with $\mathbf{i}$ replaced by $\mathbf{j}$, so solutions also exist for such indices $\mathbf{j}$.

\section{Discussion}

We shall now compare the conditions of Theorem 4.1 with various others, specialising for convenience to (1.1) with continuous $r_{m n}$. Let us start with uniform left definiteness (ULD) where it is required that the operators $T_{m}$ should be positive definite and

$$
C(x) \omega>0 \text { for all } x \in \underset{m=1}{\mathrm{X}}\left[a_{m}, b_{m}\right]
$$

for some $\omega \in \mathbb{R}^{k}$, where $C(x)$ denotes the cofactor matrix of $R(x)$. Continuity forces the components of $C(x) \omega$ to have positive lower bounds, hence the "uniformity". It can be shown [4, Lemma 2.1] that, after a nonsingular change of $\lambda$ coordinates, ULD implies

(i) $\rho_{m}^{1}(0)>0$ for all $m=1, \ldots, k$ 
(ii) $r_{m n}\left(x_{m}\right)<0$ for all $x_{m} \in\left[a_{m}, b_{m}\right]$ if $m \neq n$

(iii) $r_{m m}\left(x_{m}\right)>0$ for all $x_{m} \in\left[a_{m}, b_{m}\right]$ if $m=1, \ldots, k$

(iv) ${ }_{u} c_{m n}(x)>0$ for all $x_{m} \in\left[a_{m}, b_{m}\right], m, n=1, \ldots, k$.

It should be noted that these four conditions are not quite sufficient for the existence of eigenvalues. If

$$
\operatorname{det} R(x) \neq 0 \text { for some } x
$$

then solutions of any index are guaranteed [15, Theorem 3.5.2] but otherwise there may be no eigenvalues. As a trivial example, one could take:

\section{Example 5.1.}

$$
k=2, p_{m}=1, q_{m}=0, R(x)=\left[\begin{array}{rr}
1 & -1 \\
-1 & 1
\end{array}\right] \text {. }
$$

The two equations (1.1) then force $\lambda_{1}-\lambda_{2}$ to take opposite signs for any index. It follows that there are no eigenvalues under Dirichlet boundary conditions.

We now show that if we strengthen $(v)_{u}$ to

$(\mathrm{v})_{u}^{\prime}$

$$
\operatorname{det} R(x)>0 \text { for some } x
$$

then (i) $)_{u}$, (ii) $)_{u}$, (iv) $)_{u}$ and (v) ${ }_{u}^{\prime}$ together imply our sufficiency conditions (i), (ii), (iii) of Theorem 4.1. First, (i) $)_{u}$ strengthens (i) by requiring $\mathbf{i}=1$ and (ii) ${ }_{u}$ strengthens (ii) by requiring strict inequality (and hence a negative upper bound). Moreover, a well known result for real matrices [15, Lemma 5.5.1] shows that (iv) ${ }_{u},(\mathrm{v})_{u}^{\prime}$ imply (iii) which in the present context becomes

$$
R(x) \tilde{\mu}>0 \quad \text { for some } x \text { and some } \tilde{\mu}>0
$$

Hence we can state that, apart from (v) , which must be compatible with the initial change of $\lambda$ coordinates, our sufficient condition of Theorem 4.1 is weaker than ULD. On the other hand the following example satisfies the conditions of Theorem 4.1 but drastically fails ULD.

Example 5.2. As for Example 5.1 but with

$$
R(x)=\left[\begin{array}{cc}
\cos x_{1} & -\sin x_{1} \\
-1 & 0
\end{array}\right]
$$

on $\left[a_{m}, b_{m}\right]=[0, \pi]$ and $\mu=\left[\begin{array}{ll}-1 & 1\end{array}\right]^{T}$. It is impossible to satisfy any of (ii) ${ }_{u}$, (iii) ${ }_{u}$ or (iv) $)_{u}$ even after a change of coordinates.

Let us also point out that (i $)_{u},(\text { ii })_{u},(\text { iii })_{u}$ and $(v)_{u}$ are sufficient for the existence of 
eigenvalues of arbitrary index if $k \leqq 3$. This is trivial if $k=1$. If $k=2$ then (ii) ${ }_{u}$ and (iii) imply (iv) ${ }_{u}$ so we have a uniform left definite problem (1.1). If $k=3$ then (i) ${ }_{u}$, (ii) $)_{u}$ and (iii) ${ }_{u}$ imply local definiteness of (1.1) in the sense of [15, Theorem 3.3.2] which together with $(v)_{u}$ gives the existence of eigenvalues of any index. If $k>3$, however, then (i) $)_{u}$ (ii) $)_{u}$, (iii) ${ }_{u}$ and $(v)_{u}$ do not guarantee existence of eigenvalues.

Weaker forms of (ii) $)_{u}$, (iii) $)_{u}$ are

$$
\begin{aligned}
& \text { (ii) } r_{m n}\left(x_{m}\right) \leqq 0 \text { for all } x_{m} \text { if } m \neq n \text {, } \\
& \text { (iii) } r_{m m}\left(x_{m}\right) \geqq 0 \text { for some } x_{m}, \quad m=1, \ldots, k \text {, }
\end{aligned}
$$

which constitute Richardson's "normal form" for (1.1) (cf. [13, p. 301]) if the necessary conditions of Corollary 3.1 are satisfied with linearly indpendent $\boldsymbol{\mu}^{m}$. In this case the $\boldsymbol{\mu}^{\boldsymbol{m}}$ can be taken as $\lambda$ coordinate vectors after a nonsingular transformation. We should point out that this normal form cannot be guaranteed, since it may be impossible to choose linearly independent $\boldsymbol{\mu}^{\boldsymbol{m}}$ : cf. Example 5.2 .

Let us turn now to Richardson's Theorem [13, p. 299] which is for analytic coefficients and Dirichlet end conditions. Richardson assumes that nonzero solutions of (1.1) with $\lambda=0$ and $y_{m}\left(a_{m}\right)=0$ have $i_{m}-1$ internal zeros: this is easily seen to imply (i) of Theorem 4.1. He also assumes (3.4) and the following strengthening of (3.5):

$$
\left(R(x) \xi^{m}\right)_{m} \text { takes both signs for } x_{m} \in\left[a_{m}, b_{m}\right] \text {. }
$$

He then claims the existence of a solution to (1.1) so that each $y_{m}$ has $i_{m}-1$ internal zeros, i.e., a solution of index $\mathbf{i}$.

The following example shows that Richardson's conditions are not sufficient as stated.

Example 5.3. Let $k=3, p_{m}=-q_{2}=-q_{3} \equiv 1, q_{1} \equiv 0$,

$$
R(x)=\left[\begin{array}{ccc}
0 & x_{1} & -1 \\
\pi+x_{2} & 0 & -1 \\
-\pi-x_{3} & 0 & -1
\end{array}\right] \text { with Dirichlet conditions on }\left[a_{m}, b_{m}\right]=\left[-\frac{\pi}{2}, \frac{\pi}{2}\right],
$$

$m=1,2,3$. We choose $\mathbf{i}=1$ since each $T_{m}$ is positive definite, and with $\left[\begin{array}{lll}0 & 1 & 1\end{array}\right]^{T}$, $\left[\begin{array}{lll}1 & 0 & \pi\end{array}\right]^{T}$ and $\left[\begin{array}{ccc}-1 & 0 & \pi\end{array}\right]^{T}$ as $\xi^{m}$ we may check (3.4) and (5.1), so Richardson's conditions are satisfied. We shall prove by contradiction that no solution of (1.1) exists with index 1.

First we claim that $m=1$ in (1.1) forces

$$
\lambda_{3} \geqq-1
$$

This follows because the eigenvalue $\lambda_{3}=\lambda_{3}\left(\lambda_{2}\right)$ of

$$
-y_{1}^{\prime \prime}=\left(\lambda_{2} x_{1}-\lambda_{3}\right) y_{1}, \quad y_{1}\left( \pm \frac{\pi}{2}\right)=0
$$


corresponding to $i_{1}=1$, is a convex function having asymptotic slopes

$$
\lambda_{3}^{\prime}\left(\lambda_{2}\right) \rightarrow\left\{\begin{array}{l}
\min \\
\max
\end{array}\right\}\left(x_{1}\right)=\mp \frac{\pi}{2}
$$

as $\lambda_{2} \rightarrow \mp \infty$ : see, e.g. [5, Section 2]. Also the transformation $x_{1} \rightarrow-x_{1}$ shows that $\lambda_{3}$ is even. Hence $\lambda_{3}\left(\lambda_{2}\right) \geqq \lambda_{3}(0)=-1$ for all $\lambda_{2}$.

On the other hand, $m=2$ and 3 in (1.1) give

$$
y_{2} y_{3}^{\prime \prime}-y_{2}^{\prime \prime} y_{3}=\lambda_{1}\left(2 \pi+x_{2}+x_{3}\right) y_{2} y_{3}
$$

so integration by parts yields

$$
0=\lambda_{1} \int_{-\pi / 2}^{\pi / 2} \int_{-\pi / 2}^{\pi / 2}\left(2 \pi+x_{2}+x_{3}\right) y_{2} y_{3} d x_{2} d x_{3} .
$$

Since the integrand does not vanish, we see that $\lambda_{1}=0$. Hence

$$
-y_{3}^{\prime \prime}+y_{3}=-\lambda_{3} y_{3}, y_{3}\left( \pm \frac{\pi}{2}\right)=0
$$

and this contradicts (5.2).

We conclude by returning to Richardson's "normal form", which exists when the $\xi^{m}$ are linearly independent, as is the case in Example 5.3. In "normal form", Richardson's

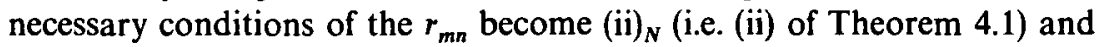

$$
\text { (iii) })_{\mathrm{RN}} r_{m m}\left(x_{m}\right)=0 \text { for some } x_{m},
$$

while his sufficiency conditions are (ii) $)_{N}$ and

$$
\text { (iii) })_{\mathrm{RS}} r_{m m} \text { takes both signs on }\left[a_{m}, b_{n}\right] \text {. }
$$

In "normal form", Example 5.3 then satisfies (i) and (ii) of Theorem 4.1, and also (iii) $\mathrm{RS}$.

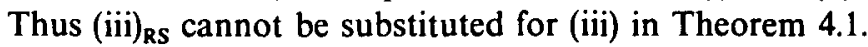

Acknowledgements. Binding thanks L. Turyn for much discussion and correspondence in the early 1980's on problems related to Richardson's. Both authors thank NSERC of Canada for supporting Volkmer's 1989 visit to the University of Calgary, during which this research was carried out.

\section{REFERENCES}

1. F. M. Arscott, Periodic Differential Equations (Pergamon Press, London, 1964). 
2. F. V. Atkinson, Multiparameter spectral theory, Bull. Amer. Math. Soc. 74 (1968), 1-27.

3. P. A. Binding, Multiparameter definiteness conditions II, Proc. Roy. Soc. Edinburgh 93A (1982), 47-61. Erratum: ibid 103A, 359.

4. P. A. Binding and P. J. Browne, Classification of eigentuples for uniformly elliptic multiparameter problems, J. Math. Anal. Appl. 139 (1989), 268-281.

5. P. A. Binding and P. J. Browne, Eigencurves for two-parameter self-adjoint ordinary differential equations of even order, J. Differential Equations 79 (1989), 289-303.

6. J. EISENFELD, On the number of interior zeros of a one-parameter family of solutions to a second order differential equation satisfying a boundary condition at one endpoint, $J$. Differential Equations 11 (1982), 202-206.

7. M. FaIerman, An oscillation theorem for a one-parameter ordinary differential equation of the second order, J. Differential Equations 11 (1972), 10-37.

8. M. Faierman, A note on Klein's oscillation theorem for periodic boundary conditions, Canad. Math. Bull. 17 (1975), 749-755.

9. W. S. Loud, Stability regions for Hill's equation, J. Differential Equations 19 (1975), 226-241.

10. J. Meixner and F. W. SchäFke, Mathieusche Funktionen und Sphäroidfunktionen (Springer, Berlin, 1954).

11. M. A. NalmaRk, Linear Differential Operators II (translated by E. R. Dawson, Ungar, New York, 1968).

12. R. G. D. Richardson, Theorems of oscillation for two linear differential equations of the second order with two parameters, Trans. Amer. Math. Soc. 13 (1912), 22-34.

13. R. G. D. Richardson, Über die notwendigen und hinreichenden Bedingungen für das Bestehen eines Kleinschen Oszillations Theorems. Math. Ann. 73 (1912/13), 289-304. Erratum: ibid. 74 (1913), 312.

14. L. Turyn, Sturm-Liouville problems with several parameters, J. Differential Equations 38 (1980), 239-259.

15. H. Volkmer, Multiparameter Eigenvalue Problems and Expansion Theorems (SpringerVerlag, 1988).

Department of Mathematics and Statistics

University OF CALGary

Calgary, Alberta

CANAdA, T2N IN4
Department of Mathematical Sciences UNIVERSITY OF WISCONSIN-MILWAUKEE P.O. Box 413

Milwaukee, Wisconsin 53201

U.S.A. 